Estudios de Psicología, vol. 24, núm. 3, 2003, pp. 261-275.

\title{
La suspensión como mecanismo de creación semiótica.
}

\section{Rivière, Angel y Español, Silvia.}

Cita:

Rivière, Angel y Español, Silvia (2003). La suspensión como mecanismo de creación semiótica. Estudios de Psicología, 24 (3), 261-275.

Dirección estable: https://www.aacademica.org/silvia.espanol/116

ARK: https://n2t.net/ark:/13683/pHOV/yMz 


\title{
La suspensión como mecanismo de creación semiótica
}

\author{
ÁNGEL RIVIÈRE Y SILVIA ESPAÑ OL ${ }^{1}$ \\ U niversidad A utónoma de $M$ adrid<smiles>C1#CC2=CCC=CC(CC1)C2</smiles> \\ Resumen
}

El desarrollo del os modos desi gnificar humano ha sido una de las preocupaciones recurrentes de Á ngel R ivière En la década de 90, una especiede cristal ización de ideas quevenía desarrollando en diferentes áreas diolugar a la elaboración de su teoria semiótica-evolutiva centrada en la idea de "suspensión semiótica". En estetexto, que seestaba escribiendo a principios de año 2000, se presenta e mecanismo desuspensi ón en toda su extensión: descri biendo detall adamente su pecul iar natural eza y fuerza semiótica; resaltando el contraste entre la diversidad demodos designificar quela suspensión logra abarcar y la simpleza del mecani smo en sí mismo; enlazando la suspensión semiótica con la génesis dela comunicación, tanto en la fil ogénesis como en la ontogéne sis, así como con el desarrollo del os etados deinter subjetividad. El texto proyecta un análisis evol utivo, punto a punto, de los procesos humanos desuspensión semiótica, de cual sepresentan sól o los dos iniciales.

Palabras dave Semiosis, significado, comunicación preverbal, intersubjetividad, ontogénesis, filogénesis, expresiones emocionales, gestos, símbolo, metáfora.

\section{Suspension as a semiotic creation mechanism}

\section{A bstract}

Thedevelopment of human ways of producing meaning was a recurrent concern in A nge R ivière's intellectual production. In the 90 's, a crystallization of ideas on which hehad been working in different real ms gave way to the daboration of his semi otic-developmental theory centred on the idea of "semiotic suspension". This paper, that was being written in the early months of 2000, describes the suspensi on mechanism at length and 1) provides a detail ed description of its particul ar natureand semi otic strength, 2) emphasizes the contrast between thesimplicity of themechanism itself and therange of types of meaning that thesuspension spans, 3) links semiotic suspension to thegenesis of communication both in phyl ogenesis and ontogenesis, as well as its rol ein the devel opment of intersubjectivity. A Ithough only thefirst two areinduded here, the paper offers a detailed deve lopmental analysis of human semiotic suspension processes.

$K$ eywords: Semiosis, meaning, preverbal communication, intersubjectivity, ontogenesis, phyloge nesis, emotional expression, gestures, symbol, metaphor. 


\section{Rarezas y paradojas de la semiótica}

¿Quérelación tienen cosas tales como la expresión amenazante del perro cuando enseña los dientes, el gesto de pedir de un niño pequeño, el juego de ficción y las metáforas poéticas más el aboradas? La semiótica, con sus raras paradojas, puede reunir en una misma mesa a tan dispares compañeros. Queremos, en este artículo, realizar una breve indagación psicosemiótica. U na exploración que recuerda mucho las sorprendentes soluciones que daba a sus problemas crimina les, para delicia de sus lectores, el - paradójicamente- cándido padre Brown, aquel entrañable personaje de Chesterton. De una reunión en que había, por ejemplo, un excéntrico millonario norteamericano, un sabio no tan distraído, el jefe de la policía de París, un cura de apariencia bobalicona, un embajador y su irresistible hija, y un apuesto teniente de la legión extranjera; de una absurda reunión imposible, en que al guien, que no podía haber entrado en la casa de ningún modo, asesinaba a otra persona que resul taba no ser el muerto que todos cre Ian que era, el buen Brown sacaba de la chistera una trama armónica que todo lo explicaba: ien realidad, el asesino era el muerto!

N osotros, con esa afición de los semióticos por descubrir nexos comunes entre sus inferencias abductivas y las de los héroes de las novel as policiacas, nos proponemos reunir también a raros compañeros de mesa y dilucidar una trama en que se reúnen episodios muy dispares en la ontogénesis, la fil ogénesis y la historia de la cultura: el cachorreo juguetón de los mamíferos que representan que luchan, el gesto de apuntar a un objeto deseado por un niño de doce meses, el trote sobre una escoba (que a su modo es un cabal lo) del niño de tres años, la metáfora poética de G arcía Lorca cuando dice que "G ranada era una luna / ahogada entre las yedras". Estas y otras muchas acciones peculiares, humanas y animales. El conejo que nosotros tenemos en la chistera, y que define un mecanismo subyacentea estas acciones dispares, no es un mal vado asesino, sino un concepto semiótico de gran potencia: la noción desuspensión.

El concepto desuspensión semióticatiene, como trataremos demostrar en este artículo, una gran fecundidad y ofrece un esquema abarcador de actividades muy diversas que constituyen diferentes modos de significar. A pesar de su potencial explicativo, es en a pariencia decepcionantemente simple. ¡Pero una precaución mínima de esos sabuesos perspicaces, que son los semióticos y los personajes tales como el padre Brown o Sherlock H ol mes, es la de no fiarse de la simplicidad apa rente delas soluciones a sus problemas! Las paradójicas simplicidades dela semiótica y la criminol ogía de ficción esconden grandes complejidades. Eso mismo sucede con el concepto de suspensión. Pero, en cual quier caso, empecemos por lo simple, por una escueta definición de diccionario: Suspender $\Theta$, en el sentido quenosotros lo decimos, "dejar al go sin fecto". H acer queuna acción, una representación del mundo o una estructura simból ica dejen de tener los efectos normales quetendrían sobre el mundo real o mental. Suspender es hacer que deje de regir al go: bien los efectos material es de las acci ones, o las propiedades literal es del mundo, o el significado aparente deun enunciado o deuna representación simbólica.

El perro que enseña los dientes, en real idad no muerde, aunque es probable que Io haga. Los cachorros que real izan una pelea lúdica, en realidad no luchan. $\mathrm{N} \mathrm{i}$ abraza el niño que levanta sus brazos para que le tomen en brazos. $\mathrm{N}$ i toca el objeto el quelo señal a para que otro lo mire. N $\mathrm{i}$ atribuye, en la "modalidad delo real", la virtud de cabal gar a las escobas el niño que monta en una. $\mathrm{N}$ i pretende García Lorca que creamos literal mente que su ciudad, G ranada, era una luna ahogada entre las yedras. N ada más enfrentarnos con ese concepto, paradójica mente simple, de "suspensión", nos encontramos con una primera idea paradójica, que no nos separa un ápice de la ilustre tradición del cura-detective de Ches- 
terton. La paradoja es la sigui ente: cuando el perro no muerde pero amenaza, el niño no toca pero señal a, el preescol ar no cabal ga real mente, o el poeta no dice - pero dice que Granada era unaluna ahogada, el mecanismo semiótico se desvela precisamente en el hueco que dejan las negaciones. En un espacio etéreo, sombreado por la huella de las acciones que no se real izan efectivamente: morder, tocar, cabal gar, afirmar literal mentela identidad entre G ranada y la luna.

Es en ese vacío, en la ausencia de efectos primeros, material es o semióticos (efectos tal es como tocar, hacer daño con los dientes o hacer creer literal mente quela ciudad andal uza es el satélite de la tierra), donde se revela paradójicamente la presencia de un mecanismo esencial de suspensión semiótica. D e un mecanismo que permite, primero, la creación de acciones semióticas a partir de acciones a secas, y luego la definición de acciones semióticas de segundo orden a partir de otras de primer orden. De forma parecida a como en el cuento del padre Brown se identificaban el asesino y el muerto, aquí hay una identidad de base (aunque expresada en niveles semióticos cada vez más poderosos y abstractos): un meca nismo que consiste en dejar al go sin efecto, en hacer que deje de regir algo, para crear significación en el espacio vacío delo queno se efectúa.

La afirmación de que hay un mecanismo subyacente común a actividades tan distantes como pedir al go extendiendo la pal ma de la mano, por una parte, y decir por otra-como hace García Lorca en el mismo poemaya citado- que "Gra nada era una corza / rosa por las veletas", no implica negar las enormes diferencias que existen entre los procesos semióticos que se ponen en juego en uno y otro caso, ni reducir los mecanismos de creación metafórica o lúdica a otros mucho más el emental es, tales como los consistentes en dej ar "a medio camino" acciones simples (como empuñar o tocar) para crear los gestos de pedir o de seña lar con función ostensiva. Como veremos a lo largo de este artículo, los mecanismos de suspensión semiótica, es decir, aquell os que consisten en crear formas de significado dejando sin efecto al go, se el aboran y complican extraordinariamente a lo largo de la ontogénesis humana. Sin embargo, una idea esencial es que esos mecanismos tienen una filiación común y se derivan, en último término, de otros mucho más primitivos que se ponen en juego en pautas de semi osis animal, incluso muy lejanas filogenéticamente de las formas más el aboradas de suspensión representacional o simbólica quese encuentran en el hombre.

\section{Algunos ejemplos de suspensión semiótica}

Con el fin de analizar el proceso de construcción ontogenética humana de formas de suspensión cada vez más complejas y de rastrear su origen animal, conviene que aclaremos primero, más y mejor, el concepto de suspensión. Para ello nos serviremos de un ejemplo especial mente obvio y revelador: el de los primeros símbol os enactivos, que comienzan a real izar los niños, con funciones comunicativas, hacia la mitad del segundo año de vida. En al gunos trabajos anteriores (Rivière, 1984, 1990, 1992), uno de nosotros ha anal izado un episodio prototípico, en que un niño de pequeño, al quellamaremos P., real iza para comunicar un deseo la siguiente secuencia de acciones:

(O bs. 1) M ientras su padre está distraído haciendo otra cosa, $P$. $(1 ; 6)$ se acerca a él con un mechero en la mano. Trata de asegurarse la atención del padre y luego, blandiendo el mechero, lo muestra y realiza varias veces la acción desoplar. Como su padre no hace caso y está distrá do, P. insistey repitela secuencia de tocar la pierna de su padre, llamarle, mostrar el mechero y soplar. Entonces, el padre deP. enciendeel mechero y el niño sonríe satisfecho.

Si entendemos, con Piaget (1959) y Bates (1979), que los símbolos son signos capaces de evocar significados ausentes, no percibidos o no visibles, mediante el 
uso de significantes claramente diferenciados; si convenimos además en que los símbol os tienen su origen en acciones externas y observables, como insisten Wolf y Gardner (1981), tendremos que aceptar que la conducta deP. incluye un núcl eo si mbólico interesante: la acción de sopl ar. Al insertar esa acción en un complejo de esquemas interactivos (que incluyen mirar, tocar la pierna, decir "ipapá!", mostrar el mechero), el niño hace un símbolo genuino, inventado por é. A similando el esquema simbólico de soplar con otro conjunto de esquemas, el niño se hace capaz efectivamente de representar al go ausente, al go quequiereque suceda pero que aún no ha sucedido. Un deseo que probablemente (y como demuestra su expresión de "haber sido entendido") puede parafrasearse así: "ipapá: quiero jugar a encender y apagar este objeto!". ¡R eal mente no hay nada más eficaz que encender un mechero para lograr inmediatamente el amor incondicional de todos los niños de 18 meses!

Pero nos interesa detenernos aquí en una pregunta: ¿qué es lo que hace que sea simból ica la acción de soplar? Es interesante destacar que esa misma acción simple, soplar, no tiene por qué serlo. Pero volvemos a encontrarnos ante uno de esos casos en que la simplicidad es engañosa. La acción de soplar, en el ejemplo propuesto, implica un mecani smo más complejo que lo que parece y, en cierto modo, extraño. El niño no sopla para extinguir una llama, como lo haría si real izara la acción instrumental de soplar. D e hecho, el mechero está apagado y el niño sopla para que su padre lo encienda. Se trata de un soplar peculiar, funcionalmente diferente a aquel que permite apagar el fuego. D eun soplar, por así decirIo, "semiótico", lanzado al vacío, suspendido; de un soplar en que se ha dejado sin efecto la función instrumental de apagar y que, precisamente por eso, toma una función semiótica. Al real izarse en suspenso, al aire, sin función de cambiar un estado de cosas en el mundo, adquiere la función semiótica de transmitir un significado a un intérprete. Precisamente porque soplar no apaga, en este caso, una Ilama inexistente, se convierte en una acción semiótica. Al desenraizarse de una de sus funciones instrumental es típicas, la acción de soplar se convierte en símbolo enactivo.

Llevarse una cucharada llena de sopa a la boca no es una acción semiótica, sino instrumental. Pero llevarlavacía - como si estuvierallena es una acción simbólica. Soplar una cerilla encendida es una acción instrumental, pero puede ser una acción semiótica soplar al aire. Galopar sobre un caballo no es, en principio, una actividad simbólica, pero cabal gar sobre una escoba suel e serlo, etcétera. Los símbol os enactivos y lúdicos de los niños pequeños manifiestan con especial cla ridad el empleo de un mecanismo semiótico que consiste en desgajar una acción de su función instrumental propia, para dar a esa acción una virtual idad representacional, sígnica. A parte de los símbolos lingüísticos, el niño desarrolla un mecanismo simbólico genuino, cada vez más el aborado, y que implica dejar en suspenso la eficiencia material de las acciones y, progresivamente, las propiedades delo real, en el caso del juego deficción.

Estas formas desuspensión, queimplican dejar sin efecto las virtualidades eficientes de acciones instrumental es 0 , en la medida en que se el aboran, las propiedades real es de los objetos, son específicamente humanas y rel ativamentetardías en la evolución ontogenética del niño (sus primeras formas el emental es se encuentran hacia los 18 meses). Si nos retrotraemos a momentos anteriores del desarrollo del niño 0, más allá aún, si nos remontamos fil logenéticamente hasta ciertas pautas de semi osis animal, encontramos mecani smos más primitivos, pero quetambién implican claramenteel empleo de procesos desuspensión.

Esto ya lo vio claramente G regory Bateson (1972) y lo planteó (aunqueno en los mismos términos) en un lúcido artículo en el que seinspira, en gran parte, la 
noción de suspensión. El artículo, "H acia una teoría de la fantasía y el juego", se detenía en la consideración de ciertas formas de semi osis animal, como las que se observan en pautas de juego de los mamíferos, en que se refleja con claridad esa peculiar "epifanía por la vía de la negación", quees una huellaclara del funciona miento del mecanismo de suspensión: "ví dos monitos jugando - comenta Bateson-, es decir, entregados a una secuencia de interacciones, en la cual las acciones-unidad o señales eran semejantes, pero no las mismas, a las del combate. Era evidente, aun para un observador humano, que la secuencia en su conjunto no era un combate, y era evidente para el observador humano que para los propios monitos participantes eso no era un "combate". A hora bien, este fenómeno, el juego, sólo podía producirse si los organismos participantes eran capaces de cierto grado de metacomunicación, es decir, de intercambiar señales quetransmitieran el mensaje: 'Esto es un juego' "(op. cit., p. 207 delatraducción española).

Es claro que esas señal es metacomunicativas, que implican una cierta capacidad de diferenciar un hecho como "puramente sígnico", como "suceso semiótico", tienen que derivarse en parte del mecanismo de suspensión de la acción. Y, en este caso, la pal abra suspensi ón tiene un sentido muy directo: se refiere, por ejemplo, al hecho de que el mordisqueo lúdico no selleva hasta el final; no es exactamente como el mordisco agónico. Es un mordisqueo a medio hacer, "suspendido". Es, si se nos permite decirlo así, un "mordisco semiótico". Y los mordiscos semióticos no hacen daño ni producen sangre. La dentellada profunda del perro quelucha no es igual quee mordisquillo suave, casi acariciante, suspendido, del perro que juega a luchar. Cual quiera que haya jugado a luchar con su perro sabrá con qué cuidado muerdeéste su brazo, hasta quépunto es del icado el cuidado que el animal tieneen "despojar de efectos" (tal es como herir y provocar sangre), en "dejar en suspenso" (como si dijéramos, "dejar sin terminar") la dentellada hiriente para convertirla en juguetona y suave caricia con los dientes.

Resulta interesante el hecho de que, al mismo tiempo que deja en suspenso la acción eficiente de morder, el ani mal que juega a luchar realiza un intenso despliegue expresivo de señal es que denotan aparente ferocidad. Y, en este caso, nos enfrentamos a otro racimo de paradojas misteriosas de la semiótica, que parecen dignas del padreBrown. En el aspecto semiótico-expresivo, la pel ea lúdica implicaun delicado equilibrio entreelementos expresivos "acentuados" y pautas deata que "amortiguadas". Pero además los el ementos expresi vos tienen una connota ción peculiar, positiva por así decirlo, muy diferente de la connotación negativa que tiene la expresividad del animal que real mentelucha y ataca. La ferocidad expresiva del perro quejuega a luchar es, en cierto modo, excesiva para ser real. Es una ferocidad juguetona, una "representación de ferocidad", que paradójicamente es "demasiado feroz" para ser auténtica. Se trata de una ferocidad positiva, por así decirlo. La delectación en los elementos expresivos de ferocidad forma parte del despliegueno-eficientede señal es quesignifican: "Esto es un juego, es un mero conjunto de signos. Lo que hago denota lucha, pero no denota lo que denotaría la lucha". Frecuentemente, los mamíferos queluchan de verdad muestran una economía expresiva mucho mayor queaquel los quejuegan aluchar.

En el artículo en que analizaba estas formas de semiosis animal, Bateson (1972) hacía un fascinante anál isis del enunciado - o la expresión metacomunicativa- "esto es un juego", destacando su carácter paradójico: "este mensaje - decía- contiene aquellos el ementos que necesariamente generan una paradoja del tipo R ussell o Epiménides, una enunciación negativa, que contiene una metaenunciación negativa implícita. Si se la desarrolla, la enunciación "Esto es un juego" suena más o menos dela siguiente manera: "Las acciones a las que esta mos dedicados ahora no denotan lo que denotarían aquel las acciones en auyo lugar 
etán'"'(p, 207). Para Bateson el carácter intransitivo dela relación de denotación, en este caso (que se refleja en la paradoja de la denotación), constituiría una proyección de un proceso de diferenciación mapa-territorio o, por decirlo más clara mente, de diferenciación de los signos en su calidad detales, de extraordinaria importancia en la evolución dela semiosis animal.

Tiene un papel decisivo en esa evolución la delimitación de marcos metacomunicativos, de marcos psicológicos que implican la suspensión de la interpretación normativa delas conductas. De forma semejante a como el observador de un cuadro cuenta con un marco material que recorta su mirada, impidiendo que se extienda el proceso de definición del objeto perceptivo y estético coherente a la pared adyacente, el animal que juega a luchar con otro deja en suspenso lainterpretación de las conductas del otro como real mente agresivas. Bateson, que rela ciona la paradoja dela denotación con un tipo de proceso mental (el "proceso primario"), en que no se establecen discriminaciones lógicas fundamental es, señala la importancia del juego en el origen de la comunicación, al ser un paso crucial en la conciencia de las rel aciones entre mapa y territorio, o entre signos y referentes: "En el proceso primario, el mapa y el territorio se identifican, en el proceso secundario pueden discriminarse. En el juego se los identifica y se los discrimina" (p. 213).

Sin embargo, los mecanismos semióticos de suspensión debieron aparecer en la filogénesis mucho antes de que se avisara cual quier clase de "proceso secundario" en el desarrollo animal del mundo mental, o cual quier atisbo de conciencia de la distinción entre signos y referentes, entre "mapas" y "territorios" en la metáfora de Bateson. Él mismo hace un comentario breve, en su artícul o sobrela fantasía y el juego, que nos poneen la pista de esa idea: "La amenaza es otro fenómeno que se asemeja al juego, en cuanto que las acciones denotan, pero son diferentes de ellas, otras acciones. El puño cerrado que se esgrime en la amenaza es diferente del puñetazo, pero se refiere a un puñetazo futuro posible (pero que, de momento, no existe). La amenaza es fácil de reconocer también entre los mamíferos no humanos" (pp. 208-209). A hora bien, en el caso de las expresiones de amenaza en mamíferos ya nos situamos ante un tipo de productos semióticos muy diferentes a las luchas lúdicas y rituales.

\section{La suspensión expresiva}

H inde (1970) ha establecido una distinción si mple y terminológicamente engañosa entre tres grandes tipos de semiosis animal: I as expresiones, las metonimias y los símbol os. Las luchas ritual es y lúdicas de los mamíferos son ejemplos prototípicos de metoni mias. La amenaza del lobo que enseña sus dientes es, en cambio, un caso paradigmático de expresión (D arwin, 1872). En realidad, el concepto de metonimia de $\mathrm{H}$ inde (tomado de la idea de aquel la figura lingüística que consisteen "tomar la parte por el todo") es, parcial mente, un sinónimo de nuestro concepto de suspensión. Un na de las formas de despojar a una acción de sus efectos (de suspenderla) consiste en iniciarla pero no terminarla, es decir, en real izarla sól o en parte. Desde este punto de vista, acciones infantiles tal es como presentar la mano extendida para pedir un objeto, levantar los brazos para ser tomado en brazos, o señalar con el dedo una cosa mostrándola, son metonimias de acciones tal es como tocar o empuñar. Eso lo veremos en su momento.

Lo que hace que sea terminológicamente equívoca la distinción de $H$ inde es el hecho de que también las emociones parecen implicar ciertos mecanismos de suspensión. En la propia obra de D arwin (1872) hay sugerencias interesantes acerca de los procesos subyacentes a las formas más el emental es de suspensión semiótica que se encuentran en las emociones. R ecordemos que D arwin pensaba 
que el hábito de expresar los sentimientos mediante ciertos movimi entos, aún cuando llegue a ser innato, habría tenido un desarrollo gradual, regido por tres principios: el Principio de los hábitosútiles asociados, el Principio dela antítesis y el Principio delas acciones debidas a la constitución del sistema nervioso.

El primero de esos principios es especial mente interesante en el contexto de nuestra reflexión sobre la suspensión: sostiene que, en ciertos estados de la mente, al gunas acciones son especial mente útiles para satisfacer necesidades, deseos, etcétera. Cuando una sensación ha conducido, durante muchas genera ciones, a un movimiento voluntario, se creará la propensión a real izar un movimiento similar ante esa sensación u otra anál oga, por muy débil que sea. H abrá una tendencia a real izar los mismos movimientos en virtud del hábito, aunque carezcan ahora de utilidad al guna. Al gunas de esas acciones podrán ser reprimidas por intervención de la voluntad, pero otras, menos susceptibles a la influencia voluntaria, pervivirán, dando lugar a movimientos que tomarán val or expresivo. Es decir, la expresión de las emociones no es una proyección del funciona mi ento de múscul os especial mente di señados para real izar movimi entos expresivos, sino reflejo de la suspensión de acciones útiles. En este caso, que constituye el ejemplo más el emental de suspensión, el mecanismo primitivo consiste en un proceso por el que se fragmenta una conducta animal, y al gunos elementos deella perviven, tomando val or designos para los congéneres.

A diferencia del juego, las expresiones de amenaza, por ejemplo, no tienen porqué implicar una intención semiótica en el organi smo que las produce. Son también previas a la aparición de la intención comunicativa, tanto en la filogénesis animal como en la ontogénesis humana. Sí tienen que tomar, desde luego, un val or de si gnos "antici patorios" para poder ser consideradas como fenómenos semióticos. Es decir, requieren un "intérprete", no necesariamente consciente de su interpretación, que actúa en consecuencia con el val or anticipatorio de esos signos. De este modo, el mecanismo de suspensión semiótica, que ya se manifiesta con claridad en las expresiones de emoción, es muy anterior a la aparición en el mundo animal de una conciencia semiótica, que parece expresarse en cambio, de forma nítida aunque no necesariamente reflexiva ni explícita, cuando aparecen metoni mias tal es como los juguetones mordisqueos de los cachorros mamíferos o las reguladas luchas ritual izadas. Conductas mediante las que los mamíferos definen relaciones, jerarquías, prioridades en el acceso a compañía sexual y otras cuestiones de una enormeimportancia social.

H emos visto hasta aquí al gunos ejemplos muy claros de suspensión semiótica: la acción de soplar al aire del niño de dieciocho meses, la de morder sin dañar del perro que juega, la de enseñar los dientes del mamífero que amenaza. Pero aún estamos muy lejos de formas muy complejas de suspensión, tal es como las que se manifiestan en el juego simbólico de los niños pequeños o en las metáforas más complejas del os poetas. Entrelos dientes agudos que enseña el perro que amenaza y la luna ahogada entrelas yedras de G arcía Lorca, parecehaber una distancia inmensa, inaccesible - isiempre está lejos G ranada!-; una distancia que, sin embargo, recorren las crías de nuestra especie en su ontogénesis, ese proceso formidable que, entre otras muchas cosas, permite la conversi ón paulatina de al gunos mamíferos en al gunos poetas. Tenemos, así, que referirnos al desarrollo humano para comprender el paso desde las formas más simples de expresión emocional animal alas más complejas desemiosis humana.

\section{El desarrollo inicial de los procesos humanos de suspensión semiótica}

Desdehaceya varias décadas el estudio dela producción de significado en psicología evolutiva se ha ido extendiendo hacia momentos cada vez más tempra 
nos del desarrollo. Se han descrito procesos semióticos preverbales intencionales como el uso de gestos (Bates, 1979) y se ha empezado a comprender que las primeras interacciones del bebé con los adultos de su entorno, que implican procesos de armonización y sintonización que se establecen desde el momento del nacimiento (Trevarthen, 1982; Rivière y Sotillo, 1999), son también intercambios de natural eza sígnica. La capacidad semiótica del bebé se desarrolla a modo un ritmo vertiginoso en sus primeros años de vida. Podríamos decir que en ese recorrido vertiginoso existen ciertos momentos puntual es que denotan cambios esenciales. Siendo al nacer un ser cuyas acciones son significativas para otros, aunque él no lo sepa ni lo pretenda, paulatinamente el bebé irá construyendo signos con laintención de producir significado en los otros.

Al principio, el bebé no produce intencionadamente sus signos. Simplemente, en la díada madreniño, se produce una interacción en la cual el recién nacido, sin tener conciencia de ello, regula mediante sus expresiones emocional es conductas y estados internos de la madre. $\mathrm{N}$ o real iza acciones que sean sígnicas desde su propia perspectiva mental, pero produce "signos para la madre". Signos quetienen consecuencias en el adul to (deahí quese haya distinguido un "estadio perlocutivo", previo a cualquier intención comunicativa, en el desarrollo de la comunicación humana), sin que impliquen ninguna clase de conciencia semióticao deintención sígnica en el bebé.

Es un hecho, hoy general mente aceptado en psi col ogía evol utiva, que los bebés cuentan, desde el nacimiento, con una dotación innata y bien diferenciada de recursos de expresión emocional, queproyectan estados internos tales como la al egría, la tristeza, la ira, el mi edo, la sorpresa, el desagrado y el interés (Ekman, 1972; Ekman y Friesen, 1971; Ekman y Oster, 1979; Izard, 1971). Las capacidades expresivas de los bebés son además mucho más complejas de lo que cabría suponer desde una observación superficial. A partir de investigaciones detal ladas sobre el Ilanto infantil, W olf (1987), por ejemplo, ha demostrado que los neonatos presentan tres tipos fundamental es de llanto, con val ores sígnicos bien diferenciados: 1.- Un llanto básico, regular y rítmico, general mente asociado al hambre; 2.- un Ilanto de cólera; 3.- un Ilanto de dolor, más irregular que el básico y con tonos más agudos. D esde la tercera semana, se añade a estos patrones uno nuevo: un Ilanto de atención. Se ha demostrado que estos patrones dellanto tienen consecuencias claras y diferenciales en las madres (si se quiere decir así, "efectos perlocutivos") y se asocian a cambios psicofisiológicos en ellas, tales como variaciones en el ritmo cardíaco y en la conductividad dérmica, siendo más inmediatas las respuestas al Ilanto de dol or queal de hambre.

Las capacidades de los adultos vincul ares para reconocer estados internos a partir de las expresiones emocionales parecen ser objetivamente muy al tas. Pero, prescindiendo de la objetividad de sus atribuciones, lo cierto es que los adultos sitúan desde el comienzo a los bebés en un contexto de interpretaciones psicológicas de gran importancia evolutiva. J ohnson et al. (1982), por ejemplo, han demostrado que las madres de niños de un mes creen reconocer, en un $99 \%$, estados internos de interés, en un $95 \%$ de al egría, en un $84 \%$ deira, en un $75 \%$ de sorpresa, en un $58 \%$ de miedo, y en un 35\% detristeza. Es interesante desta car no solo la diversidad de estados internos que reconocen los adultos sino el "sesgo positivo" de estas atribuciones (predominan estados internos positivos como el interés y la al egría sobre otros negativos, como el mi edo y la tristeza).

Al otorgar sentido eintencional idad a los signos que reciben de sus hijos, los adultos vinculares crean escenarios propicios para el desarrollo de la capacidad de generar semi osis intencionada. Algunos investigadores (Lock, 1978, y N ewson, 1978) han destacado el importante papel evolutivo que pueden tener las atribu- 
ciones, o incluso "sobreatribuciones" adultas de intención comunicativa en el desarrollo de pautas real mente intencionadas de comunicación en los bebés, al comenzar el último trimestre del primer año de vida. En la interpretación de estos autores, los bebés Il egan a comunicarse de forma intencionada (mediante signos suspendidos o no) precisamente porque sus conductas han sido considera das como intencionadas y humanamente significativas desde el inicio, cuando aún no eran producto de intenciones: "las acciones de los bebés .dice-N ewsonno sólo se reflejan de forma automática en términos de sus consecuencias físicas sino que son sometidas desde el principio a un filtro subjetivo de interpretación humana, detal forma que al gunas, pero sólo al gunas, de esas conductas son consideradas como rel evantes y coherentes en términos humanos: se juzgan como movimientos que se derivan de intenciones o comunicaciones (o bien comunica ciones potencial es) dirigidas a un "otro" social menteimplicado (...) Precisamente porque las madres atribuyen sentido a las conductas de sus bebés, éstas llegan a ser significativas desdeel punto de vista de los propios bebés" (1978, p. 37).

Es probable que el desarrollo de capacidades de semiosis intencionada en los bebés no sea sólo un producto social de las sobreatribuciones adultas, sino que se derive de un proceso complejo de interacción entre el organismo y el ambiente, en que también tienen un papel importante las capacidades innatas de madura ción cerebral. En todo caso, resulta interesante observar quéacciones en concreto son las que tienden a ser interpretadas como social mente relevantes, o como comunicativas, por parte de los adultos que rodean al bebé. D esde pocas semanas después del nacimiento, los bebés expresan con claridad patrones diferenciados de activación, atención y respuesta antelas personas y las cosas. Se han definido, por ejemplo, pautas de "preal cance", consistentes en movimientos de apertura y cie rre de la manos, y que tienden a ser suscitadas por objetos interesantes, frente a movimi entos faciales, que incluyen por ejemplo acciones de abrir y cerrar la boca, y que han recibido el nombre de "protogestos", que se dan preferentemente en respuesta a las personas interesantes. Son estos protogestos, sobre todo, los quetienden a ser interpretados como dotados de intención comuni cativa y como pautas relevantes en cursos de interacción humana(Trevarthen, 1982).

Es evidente que los protogestos y los movimientos de preal cance son patrones expresivos suspendidos, que expresan un núcleo primitivo de diferenciación, muy precoz, de dos mundos diferentes en la mente del niño: un mundo físico y otro social. En su desarrollo, el bebé tendrá que descubrir posteriormente que el primero se rige por principios de causación eficiente y el segundo por principios de causación teleologica, intencional. Que sólo en éste, y no en aquel, es virtualmente eficaz la influencia semiótica. En los primeros meses de vida, no hay evidencias empíricas sólidas que permitan atribuir aún al bebé una conciencia mínima dela distinción de un mundo intencional (vid., sin embargo, Premack y Premack, 1995), aunque sean numerosos los datos que indican que esa distinción es muy precoz en el desarrollo (Gelman, Durgin y K aufman, 1995; Premack y Premack, 1995; Spelke, Phillips y W oodward, 1995) y está nítidamente establecida en los últimos meses del primer año, cuando ya han aparecido patrones de suspensión semiótica queimplican formas de comunicación intencional.

La constitución delas primeras formas de comunicación intencional humana, que - como luego veremos- se basan en mecanismos de "suspensión metonímica", implica una cierta forma primaria de conciencia semiótica, que es como decir "conciencia del otro como intérprete" o "conciencia del otro como sujeto". Es decir, en el caso de nuestra especie al menos, la intención comunicativa (que aún no existe en el bebé de dos meses, pero sí en el de diez o doce) se corresponde con el desarrollo de formas complejas de intersubjetividad. Formas que conlle 
van, sin duda, la posesión de una cierta "noción del otro como sujeto", y de una capacidad de suscitar intencionadamente condiciones que permiten compartir estados mental es. $\mathrm{N}$ o olvidemos que, desdeel origen mismo dela comunicación humana, aparecen pautas de relación comunicativa que no sól o tienen la finalidad de "cambiar el mundo físico a través del otro" (patrones protoimperativos), sino también la de "compartir el mundo mental con el otro" (patrones protodeclarativos), como está claramente demostrado al menos desde los trabaj os de Bates (1976) y, mucho antes, en Stern y Stern (1909) o Bühler (1918).

Se plantea, en este caso, un tema de capital importancia para comprender el desarrollo de la semiosis humana y animal. $N$ os referimos al problema de la relación entre las capacidades semióticas y las competencias deatribución mental ista e intersubjetiva en el mundo animal. En éste, hay un momento decisivo en el desarrollo de la competencia de producir signos: aquel en que el organismo que los produce se hace, por así decirlo, consciente de estar produciendo una actividad meramente sígnica y que, por ende, no ejerce efectos materiales sobre el mundo, sino que tiene consecuencias mental es en los compañeros de interacción. Probablemente es ese el momento en que es posible empezar a hablar de un mundo de "significados" en la mente animal. El desarrollo de esta idea exige reformular, en térmi nos interaccionistas, la noción de significado, una pretensión teórica hercúlea, pero que aún reserva muchas sorpresas. Es muy probable que, a largo plazo, sólo sea posible comprender en profundidad esa noción central de la semiótica, la noción designificado, partiendo de la idea de quel os significados son siempreproductos genéicos de relaciones y no pueden comprenderse como subproductos dementes queactúan en soledad.

Pero los problemas que se acumulan en la mesa del investigador cuando atisba esa nueva noción de significado son enormes. Por ejemplo: es evidente que hay formas humanas de semiosis (desde los nueve o diez meses, y con una elaboración rápida en el desarrollo del niño), pero también formas animales de semi osis, que parecen rel acionarse deforma muy íntima con una "conciencia semiótica", con la pretensión de influir en estados internos ajenos mediante signos. Así, no parece posible la producción, ni siquiera implícita y metacomunicativa, de lo quellamaremos el mensaje de B ateson, a saber: "Esto es un signo, esto es un juego por ejemplo. Denota al go pero no denota lo que denotaría ese al go", sin al guna concienda, aunquesea implíata, no verbal, y pre rélexiva, dela existencia de mundos mentales en los congéneres o compañer os de interacción, de mundos regidos por principios de causación intencional, y no meramente eficiente.

¿Existe entonces al guna clase de conciencia de ese tipo en los mamíferos que, como hemos visto, "fingen" en el juego o real izan pautas metonímicas de relación? ¿Saben implícitamente los perros quejuegan que sus compañeros de juego son sus intérpretes? ¿En qué puede consistir ese saber? Y, en un sentido más teórico, ¿no implica esa idea que la constitución de un mundo de significados es, desdeel inicio, una actividad intersubjetiva? ¿Q ué decir entonces de los model os "solipsistas" y agenéticos del significado, que han tenido tanta fuerza en la psicología cognitiva reciente?

En cualquier caso lo que está claro es que, en el diseño del desarrollo humano, el desarrollo de la conciencia y la habilidad semiótica se anuda, de forma inextricable, con el desarrollo de la "conciencia del otro", de la intersubjetividad si se quiere decir así. Por eso tiene especial importancia, en ese desarrollo, la aparición, ya desde el segundo o tercer mes de vida, de patrones de interacción entre los bebés y los adultos vinculares que claramente expresan la existencia, en los primeros, de pautas de "intersubjetividad primaria", brillantemente demostradas por Colwin Trevarthen (1982). Los complejos, y afinadamente coordinados, 
intercambios de expresiones entre las madres y los bebés de dos o tres meses han sido analizados por este investigador, quellega a la conclusión de quelos copartícipes de esas interacciones mantienen relaciones "protoconversacional es", indicativas de una motivación esencial e innata de "compartir y entenderse", por parte del bebé, y de capacidades iniciales de acceso primario, expresivamente coordinado, al mundo mental del otro en el bebéhumano.

Sin embargo, estas formas primarias de intersubjetividad, que se manifiestan empíricamente en la producción, por parte del bebé, de abundantes y aj ustados gestos expresivos que son "especulares o complementarios" de los de la madre (Trevarthen, op. cit.) no parecen implicar aún ninguna conciencia, ni siquiera implícita, del otro como sujeto, ni la existencia de una "intención comunicativa" en sentido estricto. Hasta el último trimestre del primer año de vida no se manifiestan en el niño las primeras pautas de relación intencionadamente comunicativas. Los primeros atisbos de el las (hacia los nueve meses) inauguran una fase diferente del desarrollo, a la que Bates (1976) ha denominado "fase il ocutiva", en la que el bebé va a desarrollar rápidamente tanto sus habilidades como sus intenci ones de comuni carse. Con la aparición de las primeras pautas estrictamente comunicativas, que se manifiestan en los patrones protoimperativos y protodeclarativos de los bebés, encontramos que se produce un cambio importante y significativo en los mecani smos de suspensión. A parecen, en el hombre, formas peculiares de suspensión metonímica que merecen un análisis detallado.

\section{Conducta comunicativa y suspensión de contacto}

En el cuarto estadio del desarrollo sensoriomotor, entre los nueve y doce meses, se producen cambios importantes en la mentedel niño. En L a naissancede I'intel ligencechez I'enfant, Piaget (1936) comenta que es en ese momento del desa rrollo cuando se observa la aparición de conductas verdaderamente intel igentes, y reguladas por una genuina dirección intencionada. En el caso de las rel aciones con las personas, esa transformación de la conducta infantil tiene una significa ción especial, desdee momento en queimplicala aparición de ciertas conductas de interacción que implican ya una intención comunicativa. D esde los estudios clásicos de Bates (1976), cientos de investigaciones se han dedicado a anal izar, observar y comprender el fascinante desarrollo de esas primeras pautas de comunicación, que implican acciones tales como señal ar con el dedo un objeto con la finalidad de compartir el interés en él, alargar el brazo con la mano extendida para obtenerlo a través de los compañeros de interacción, o levantar los brazos para ser tomado en brazos por ellos.

Se han diferenciado varias funciones comunicativas en esas primeras conductas de rel ación guiada por intenciones claras, quetienen los bebés. $H$ ay dos de ellas que tienen una importancia esencial, y que han dado nombre a las pautas Ilamadas protoi mperativas y protoded arativas (también, "ostensivas"). Son, en realidad, los núcleos germinal es de la mayor parte de las complejas y delicadas funciones ilocucionarias que el hombre cumple por medio de la comunicación. Las primeras, las pautas protoimperativas, implican intentos de lograr cambios en el mundo a través de las personas. Las segundas, las protodeclarativas, parecen tener una intención por así decirlo más "desinteresada" y genuinamente social: parecen buscar compartir la atención y el interés por los estados del mundo. Así, cuando un bebé levanta sus brazos para ser tomado en brazos, real iza un gesto protoi mperativo. Cuando muestra a la madre un objeto atractivo, señal ándolo con el dedo, sin otra finalidad que compartir el interés por el objeto, hace un gesto protodeclarativo. 
U na idea semióticamente profunda es la siguiente: la intención comunicativa de los gestos protoimperativos (cuya final idad es pedir) se satisface en la medida en que se producen los cambios en el mundo que pretenden. Pero, en rigor, la intención comunicativa de las pautas ostensivas, sólo se satisface en la mente de los otros y en la medida en que se producen en ella procesos que implican compartir intersubjetivamente intereses. Dicho de un modo simple, pero muy claro: los protoimperativos buscan, en último término, cambiar el mundo físico (o pretenden hacerlo), los protodeclarativos el mental. Ello implica también la idea de que los requisitos intersubj etivos necesarios para la producción de conductas ostensivas son mucho más exigentes que los necesarios para las conductas protoimperativas. N o es extraño así que las personas con espectro autista tengan muchas más dificultades para desarrollar funciones ostensivas que para adquirir, por aprendizaje, funciones imperativas (Curcio, 1978; R ivière, 1997; W etherby, 1986).

Parece evidente en efecto que, para el desarrollo de pautas protodeclarativas de comunicación, se requiere un cierto grado de elaboración consciente y conceptual de la intersubjetidad primaria de que hablábamos en el apartado anterior. Ya no basta con queel niño sea capaz de compartir, sin mediaciones conceptuales, estados emocional es a través del intercambio de gestos expresi vos. Es necesario que tenga al guna clase de noción de primer orden de que el otro es un sujeto (si se quiere decir así, una "percepción", cuando menos, del otro como sujeto). Es decir, un intérprete, un ser dotado de una cierta interioridad mental. Sólo así puede explicarse que trate de compartir con el otro la atención y el interés por los objetos. El hecho de que las pautas protodeclarativas sean específicas del hombre, y no se encuentren ni siquiera en los mamíferos filogenéticamente más cercanos aél, pareceir en contra de nuestra sospecha anterior dela existencia de al guna clase de conciencia semiótica en animales no humanos, pues parece indudable que la noción de conciencia semiótica (aunque ésta no sea explícita sino implícita) es correlativa a la de inter subjei vidad secundaria. Es decir, a la de una forma de intersubj etividad, que se desarrolla desde los ocho o nueve meses de formarápida(Trevarthen y Hubley, 1978).

Cuando nos enfrentamos al problema de explicar la génesis de esas pautas protoimperativas o protodeclarativas, tal es como señalar con el dedo cosas intere santes, pedir con la mano extendida al go o sol icitar ser tomado en brazos ajenos extendiendo los propios, nos encontramos con claridad ante nuestro ubicuo compañero: el concepto de suspensión. Al fin y al cabo, esos gestos para pedir y mostrar no son sino tipos peculiares de metonimias animales. Es decir, implican el empleo de los mecanismos de suspensión, de "dejar sin efecto al go", que consisten en tomar la parte por el todo. En real izar sólo una parte de una conducta total, dejándola iniciada, "en el aire". ¿Qué conductas "totales" deja en el aire el niño cuando apunta con el dedo a un juguete, mientras mira al ternativamentea éste y a la madre, o cuando lo pide, extendiendo el brazo y la mano hacia él? En un lúcido análi isis dela "I nternal ización de las funciones superiores", Lev Vygotski (1930; ed. esp. de 1979) anticipaba genial mente la idea de que los primeros gestos comunicativos del niño resul tan de la suspensión de acciones tales como empuñar o tocar las cosas. El lector perdonará que incluyamos una larga cita de Vygotski, en que se formula con toda cl aridad la idea de suspensión y dela géne sis de los gestos comunicativos en acciones queno lo son en origen

"Llamamos internalización - diceVygotski- a la reconstrucción interna de una operación externa. Un buen ejemplo de este proceso podríamos hal larlo en el desarrollo del gesto de señalar. Al principio, este ademán no es más que un intento fallido de al canzar al go, un movimiento dirigido hacia un cierto objeto 
que designa la actividad futura. El niño intenta al canzar un objeto situado fuera de su al cance; sus manos tendidas hacia ese objeto, permanecen suspendidas - $\mathrm{el}$ subrayado es nuestro- en el aire. Sus dedos se mueven como si quisieran agarrar al go (...). Cuando acude la madreen ayuda del pequeño y se da cuenta de que su movimiento está indicando al go, la situación cambia radical mente. El hecho de señalar se convierte en un gesto para los demás. El fracasado intento del niño engendra una reacción, no del objeto que desea, sino de otra per sona. Por consiguiente, el significado primario de este fracasado movimiento de apoderarse de al go queda establ ecido por los demás. Ú nicamente más tarde, cuando el niño es capaz de relacionar su fall lido movimiento de agarrar con la situación objetiva como un todo, comienza a interpretar dicho movimiento como acto de señalar. En esta coyuntura, se produce un cambio en la función del movimiento: de un movimiento orientado hacia un objeto se convierte en un movimiento dirigido a otra persona, en un medio de establecer relaciones. EI movimiento de asir se transforma en el adto deseñalar" (1930, p. 93 en la edición españolade 1979).

Con independencia de que sea o no correcta la hi pótesis de que es el intento "fracasado" y reinterpretado por el adulto el que "fija" la suspensión como fenómeno semiótico, al ser comprendida la secuencia completa por el niño, lo cierto es que Vygotski se convierte, en esta cita, en otro ilustre precursor de la idea de que un mecanismo básico de semiosis es el que consiste en dejar en suspenso un movimiento o una acción, sustituyendo su virtualidad efectiva por un val or sígnico. Además, intuye muy perspicazmente una idea poderosísi ma: Ia de que todo ese proceso es un componentegenético esencial de los importantes procesos de internalización que se llevan a cabo en el desarrollo humano. Y menciona, a nuestro entender correctamente, un ejemplo claro de una de las "fuentes" o acciones-origen que se dejan en suspenso característicamente en los primeros gestos comunicativos intencional es de los niños pequeños: "el movimiento de asir".

Creemos, en efecto, que los primeros gestos comunicati vos de los niños pequeños se derivan de ciertos tipos de acciones, como el movimiento de asir, a las que daremos el nombre de pre acciones. Las Il amamos así porque se trata de acciones, tal es como empuñar, agarrar, asir o tocar, que normal mente son condiciones de posibilidad para otras acciones. N o son propiamente acciones instrumentales, sino condiciones o requisitos para que se realicen esquemas sensoriomotores simples o esquemas instrumentales. A sí normal mente los niños no cogen o tocan un objeto porque sí, sino quelo hacen para llevarlo a la boca, sacudirlo, restregarlo o emplearlo con fines instrumental es. En definitiva, la tesisque sostenemos es que las primeras pautas comunicativas intencional es se producen mediante el mecanismo semiótico de suspensión de preacciones (como asir 0 tocar). D iremos en este caso que la fuente de suspensión es una preacción y el producto semiótico es un gesto comunicativo, un signo que aún no tiene carácter simbólico (y un buen reflejo de ello es que no puede remitir a objetos ausentes. ¡N o se puede señal ar lo ausente estirando el dedo índice, ni pedirlo presentando una mano extendida!).

Se ha discutido mucho qué tipo de preacciones podrían constituir las fuentes de las que se derivaría por suspensión el gesto de señal ar, muy prototípico del hombre y muy ligado a su específica función comunicativa de proto / declarar. En los años setenta y ochenta, se aceptaba general mentela génesis propuesta por Vygotski, según la cual esquemas pragmáticos no comunicativos se convertirían en signos, al ser "intervenidos" interactivamente por los adultos (Lock, 1980; Clark, 1978). Pero ya en los años sesenta, W erner y K aplan (1963) habían afirmado queel origen del gesto de señal ar no se encuentra en el acto de al canzar un 
objeto (un acto ligado al mundo pragmático dela acción), sino en el acto de tocarlo, más relacionado con el mundo de los objetos contemplados o explorados. Bates retomó esa idea en su trabajo clásico sobre la génesis de la comunica ción en el niño (Bates, 1976). Pero, con independencia del carácter "pragmático" 0 "contemplativo" de los actos, lo cierto es que se aceptaba general mente la idea de que el origen de señalar reside, en nuestra terminología, en la suspensión metonímica de preacciones. También se ha implicado un mecanismo de imitación (K aye, 1982; Carpenter, N agell y Tomasello, 1998) en el origen del señal ar. Para complicar aún más las cosas, Butterworth (1998), aun aceptando la idea de que el gesto de señal ar se deriva del acto de asir, defiende que el proceso por $\mathrm{e}$ que se produce esa derivación no es el de suspensión, o ritualización, sino el proceso deantítesis propuesto por $\mathrm{D}$ arwin (1872) ....

\title{
N otas
}

\begin{abstract}
${ }^{1}$ Estees un trabajo que quedó inconcluso como consecuencia dela súbita muertedeÁngel Rivière, publicándose interrumpido, tal como entonces quedó. A parece aquí por primera vez en su versión castellana original. Una versión italiana de estemismo trabajo, a la que se agregó la bibliografía que aquí se incluye, apareció bajo el título de Rivière, A. y Español, S (2002). La sospensione come meccanismo di creazione semiotica. En G. Padovani y F. Braga Illa (Eds).R appresentazione e teorie della mente. (pp. 1-24). Parma: MonteU niversità ParmaEditore.
\end{abstract}

\section{Referencias}

BATES, E. (1976). L anguageand C ontext: T heacquisition of pragmatics. N uevaY ork: A cademic Press.

BATES, E. (1979). T heemergence of symbol s: C ognition and communication in infancy. N uevaY ork: A cademic Press.

BATESON, G. (1972). Steps to an E cology of M ind. Aylesbury: Chandler. (V.O.: Bateson, G. A theory of play in fantasy. A .P.A . P sychiatric R esearch R eports, 2, 1955).

BÜHLER, K. (1918). D iegejstigeE ntwicklung der Kinder. Leipzig: H irzel. [Trad. cast. (de la 6ạ ed. al emana) de R osario Fuentes: El desarrollo espiritual de niño. Madrid: Espasa Cal pe, 1934].

BUTTERW ORTH, G. (1998). Origins of social visual attention in infancy. M onographs of the Society for Research in Child D evelopment, $225,63(4), 144-166$.

Carpenter, M., N Agell, K .\& TOMAsello, M. (1998). Social cognition, joint attention, and communicative competence from 9 to 15 months of age. M onographs of the Society for Research in C hild D evelopment, 225, 63 (4).

CLARK, R. A. (1978). The transition from to action to gesture. En A. Lock (Ed.), A ction, gesture and symbol: the emergence of language (pp. 231-257). Londres: A cademic Press.

CuRCIO, F. (1978). Sensoriomotor functioning and communication in muteautistic Children. J ournal of A utism and Childhood Schizophrenia, 8, 281-92.

DARW IN , CH . (1872/1998). La expresión delas emociones en los animales y en el hombre Madrid: Alianza.

EKMAN, P. (1972). Universal and Cultural Differences in Facial Expressions of Emotion. En J. K. Cole (Ed.), N ebraska Syposium on M otivation, 1971. Lincoln, NE: U niversity of N ebraska Press.

EKMAN , P. \& FRIESEN, W . (1971). Constants across cultures in the face and emotions. J ournal of Personality and Social Structure, 17, 124-129.

EKMAN , P. \& OSTER, H. (1979). Facial expressions of emotion. A nnual R eview of P sychology, 30, 527-554.

GELMAN, R., DURGIN , F. \& K AUFMAN, L. (1995). Distinguishing between animates and inanimates: N ot by motion al one En D. Sperber, D. Premack \& A. J. Premack (Eds.), C ausal cognition: A multidisciplinary debate Symposia of the Fyssen Foundation (pp.150-184). N uevaY ork: Clarendon Press/Oxford U niversity Press.

H IN DE, R. A. (1970). B iological basis for human communication. N ueva Y ork: M cG raw-H ill. (T rad. cast.: B ases biológicas dela conducta social humana. Madrid, Siglo XXI, 1997).

IZARD, C. E. (1971). Theface of emotion. N uevaY ork: A ppleton-Century-Crofts.

KAYE, K. (1982). TheM ental and social life of babies. H ow parents create persons. Chicago. The U niversity of Chicago Press (Trad. cast.: L a vida mental y social del bebé Barcelona: Paidós 1986).

Lock, A. (1978). The emergence of language. En A. Lock (Ed.), A cion, getureand symbol: the emergence of language (pp. 3-18). Londres: A cademic Press.

Lock, A. (1980). T heguided rénvention of language. Londres: A cademic Press.

N EW SON, J. (1978). Dialogue and development. En A. Lock (Ed.), A ction, gestureand symbol: the emergence of language (pp. 31-43) Londres: A cademic Press.

PIAGET, J. (1936). L a naissancedel'intel ligencechez l'enfant. París: Delachaux et N iestlé (Trad. Cast.: El nacimiento delainteligencia en el niño. Madrid: A guilar, 1969).

PIAGET, J. (1959). L a formation du symbol echez I'enfant: I mitation, jeu et rêve I mage et representation. N euchâtel: Delachaux et N iestlé (Primer edición 1945) (Trad. cast.: L a formación de símbol o en el niño. I mitación, juego y sueño. I magen y representación. M éxico. Fondo de Cultura Económica)

Premack, D. \& Premack, A.J. (1995). Intention as psychological cause. En D. Sperber, D. Premack \& A.J. Premack (Eds.), C ausal cognition: A multidisaplinary debate Symposia of theFyssen Foundation (pp. 185-199). N uevaY ork: Clarendon Press/Oxford University Press. 
Rivière, A. (1984). Acción einteracción en el origen del símbolo. En J. Palacios, A. Marchesi \& M. Carretero (Comps.), P sicol ogía E volutiva. Vol 2. D esarrollo cognitivo y social de niño (pp. 145-174). Madrid: Alianza.

Rivière, A. (1990). Origen y desarrollo dela función simbólica en el niño. En J. Palacios, A. Marchesi \& C. Coll (Comps.), D esarrollo psicológico y educación (pp. 113-130). Madrid: Alianza.

Riviėere, A. (1992). Lenguajey símbolos: la dimensión funcional. En M. Belinchón, J. M. Igoa \& A. Rivière, P sicol ogía del lenguaje Investigación y teoría (pp.181-234). Madrid: Trotta.

Rivière, A. (1997). Teoría della mente e metarappresentazione. En F. Braga Illa. (Ed.), L ivelli di rappresentazione (351-410). U rbino: Quattro venti.

Rivière, A. \& Sotillo, M. (1999). Comunicazione, sospensione e semiosi umana: le origini della pratica e della compresione Enterpersonal i. Ricerchedi sociol ogia epsicologia della comunicazione, 1, 45-76.

SPELKE, E. S., PHILLIPS, A. \& W OODW ARD A. L. (1995). Infants' knowledge of object motion and human action En D. Sperber, D. Premack \& A. J. Premack (Eds.), C ausal cognition: A multidisciplinary debate Symposia of the Fyssen Foundation (pp.44-78). $\mathrm{N}$ uevaY ork: Clarendon Press/Oxford University Press.

Stern, C. \& STERN, W . (1909/1931). M onographien über die sedischeEntwickhung des Kindes. Vol 2: E rinnerung, A ussageund L ügein der ersten Kindheit. Leipzig: Barth.

TREVARTHEN, C. (1982). The primary motives for cooperative understanding. En G. Butterworth \& P. Light (Eds.), Social Cognition: Studies of thedevelopment of understanding (pp. 77-109). Brighton: H arverster.

TREVARTHEN , C. \& H UBLEY, P. (1978). Secondary intersubjectivity: confidence, confiding and acts of meaning En the first year. En A. Lock (Ed.), A ction, gestureand symbol: theemergence of language (pp. 183-229). Londres: A cademic Press.

VYGOTSKI, L. (1930/1979). EI desarrollo del os procesos psíqui cos superiores. Barcel ona: Grijal bo.

W ERNER, H. \& K APLAN, B. (1963/1984). Symbol formation. H ill sdale, N J: LawrenceErlbaum A ssociates Inc.

W ETHERBY, A. M. (1986). Ontogeny of communicative functions in autism. J ournal of A utism and D evelopmental D isorders, 16, 295-316.

W OLF, D. \& GARDNER, H. (1981). On the structure of early symbolisation. En R. L. Schiefelbusch \& D. D. Bricker (Eds.), Early language A cquisition and intervention (pp. 287-327). Baltimore: University Park Press.

W OLFF, P. H. (1987). T heD evelopment of B ehavioral States and theexpression of E motions in Early Infancy: N en Proposal s for Investigation. Chicago: University of Chicago Press. 